\title{
A meta-analysis of the prognostic significance of Golgi protein 73 in hepatocellular carcinoma in Chinese patients
}

\author{
Jian Zhang ${ }^{1}$, Manka Zhang ${ }^{1}$, Huimin $\mathrm{Ma}^{2}$, Xincheng Song ${ }^{1}$, Lingling $\mathrm{He}^{3}$, Xiaohui Ye ${ }^{4}$, Xin $\mathrm{Li}^{1,2}$
}

\author{
${ }^{1}$ Department of Center of Integrated Traditional Chinese and Western Medicine, \\ Peking University Ditan Teaching Hospital, Beijing, China \\ ${ }^{2}$ Department of Center of Integrated Traditional Chinese and Western Medicine, \\ Beijing Ditan Hospital, Capital Medical University, Beijing, China \\ ${ }^{3}$ Department of Institute of Infectious Disease, Beijing Ditan Hospital, Capital Medical \\ University, Beijing, China \\ ${ }^{4}$ Department of Institute of Infectious Disease, Peking University Ditan Teaching \\ Hospital, Beijing, China
}

Submitted: 19 November 2018

Accepted: 2 February 2019

Arch Med Sci 2020; 16 (5): 1104-1110

DOI: https://doi.org/10.5114/aoms.2019.83821

Copyright $\odot 2019$ Termedia \& Banach

\section{Abstract}

Introduction: In recent years, an increasing number of studies have revealed the possible prognostic significance of Golgi protein 73 (GP73) in hepatocellular carcinoma (HCC), but the results are still controversial. Therefore, we performed a meta-analysis to explore the possible correlation between GP73 and prognostic value in HCC.

Material and methods: Relevant publications were searched for in PubMed, EMBASE, Cochrane Library and the Chinese Biomedical Literature Database up to March 2018. Odds ratios (ORs) or hazard ratios (HRs) and $95 \%$ confidence intervals $(\mathrm{Cl})$ of eligible studies were assessed by either fixed-effect or random effects models. Publication bias analysis was also performed to assess the reliability of the meta-analysis results.

Results: In total, 9 studies including 1292 patients with HCC were included and analysed systematically in the study. The results indicated that GP73 overexpression was significantly associated with later tumour stage, higher tumour grade and poor overall survival (OS). Combined analysis of three studies showed no statistical correlation between high GP73 expression and disease-free survival (DFS). Subgroup analyses were also performed to illustrate the relationship between high GP73 expression and OS.

Conclusions: The meta-analysis suggested that overexpression of GP73 may be associated with poor prognosis in HCC and may also have a predictive role for HCC invasion and metastasis.

Key words: GP73, HCC, prognosis, meta-analysis.

\section{Introduction}

Hepatocellular carcinoma ( $\mathrm{HCC}$ ) is the sixth most common tumour and the third leading cause of cancer mortality globally [1-4]. Surgical resection and liver transplantation combined with systemic therapy is considered an effective treatment for HCC, but the 5 -year survival rate is still below $12 \%[5,6]$. Therefore, it is urgent to fully understand the pathogenesis of HCC and explore effective biomarkers that can predict

\author{
Corresponding author: \\ Xin Li \\ Beijing Ditan Hospital \\ Capital Medical University \\ 8 Jing Shun East St \\ Beijing 100015, China \\ Phone: +8684322130 \\ Fax: +8684322146 \\ E-mail: leaxin@sina.com
}


the progression and prognosis of patients with HCC after clinical treatments, which may provide doctors a novel and effective treatment strategy and improve the survival rate of HCC patients.

GP73, a type-II Golgi transmembrane glycoprotein, was first reported in 2000 [7]. Many studies have shown that GP73 is highly expressed in HCC but poorly or not expressed in normal adult tissue [8]. A number of previous publications suggested that GP73 can be regarded as a reliable diagnostic biomarker [9-11] and that its diagnostic sensitivity and specificity may be superior to those of $\alpha$-fetoprotein (AFP) [12-14]. Further research showed that GP73 expression was positively associated with HCC invasion and metastasis [15-17]. GP73 may be a new and independent biomarker for evaluating the prognosis of HCC patients. However, the results of different articles regarding GP73 in predicting the prognosis in HCC are still controversial. Hou et al. [18] conducted a meta-analysis previously to assess the relationship of GP73 expression and patients' prognostic statuses after surgery. However, Xu Hou's study evaluating the prognostic value of GP73 in patients with HCC included only 5 studies. The most recently published studies that explore the correlation between GP73 and prognostic value in HCC were not incorporated $[15,17,19,20]$, restricting the statistical power of the meta-analysis. Therefore, we performed a meta-analysis to comprehensively and systematically evaluate the association between GP73 expression and HCC prognosis.

In this meta-analysis, we aimed to evaluate the correlation of GP73 expression with OS and DFS in HCC with the available data extracted and pooled from included studies. In addition, we analysed the correlation between GP73 expression and clinicopathological parameters (tumour stage and grade).

\section{Material and methods}

\section{Literature search}

PubMed, EMBASE, Cochrane Library and the Chinese Biomedical Literature Database were searched systematically in March 2018 to identify eligible studies. The search keywords were ("Golgi protein 73" [All Fields] OR "GP73" [All Fields]) AND ("hepatocellular carcinoma" [All Fields] OR "HCC" [All Fields]) AND ("prognosis" [All Fields] OR "prognostic" [All Fields]).

Eligible studies had to meet the following criteria: (1) hazard ratio (HR) and 95\% confidence interval (CI) for OS and DFS were provided or could be calculated from available information; (2) the sample size was more than 20; (3) the level of GP73 was measured by immunohistochemistry; and (4) the study was published in English or Chinese.

\section{Data extraction and quality evaluation}

Data from each article were extracted independently by two investigators (Jian Zhang and Manka Zhang) based on the standard forms. All authors of the study discussed the differences if the two investigators had a disagreement. The following information was recorded from each study: first author, sample size, follow-up periods, method of clinical treatment, publication year, patients' basic features, cut-out level, vascular invasion, hepatic cirrhosis, the differentiation and stage of tumour, HR or OR and its $95 \% \mathrm{Cl}$. If the studies included in the meta-analysis did not provide HRS and their 95\% Cls directly, the data were extrapolated indirectly by methods introduced by Tierney et al. [21] or a Kaplan-Meier survival curve or the author was contacted to obtain the raw data.

A quality assessment was also evaluated by two independent investigators who applied the standard Newcastle-Ottawa scale, which included 3 factors: Selection, Comparability, and Outcome categories. Numbers 0 and 9 (labelled with stars) represent the lowest and highest quality, respectively. Those with 6 or more stars were graded as high-quality studies.

\section{Statistical analysis}

In this meta-analysis, Review Manager 5 and IBM SPSS Statistics 20 were applied to conduct all statistical analyses (available from http://www.cochrane. org/). Hazard ratios (HR) and 95\% confidence intervals $(95 \% \mathrm{Cl})$ were determined to assess the associations of GP73 expression with overall survival (OS) and disease-free survival (DFS). Odds ratios (ORs) with $95 \% \mathrm{Cls}$ were conducted to evaluate the relationship between GP73 and clinicopathological features for HCC. The $R^{2}$ statistic was adopted to evaluate the extent of heterogeneity. If the $l^{2}$ value exceeded $50 \%$, then significant heterogeneity was present, and the random effects model was adopted [22]. Otherwise, a fixed-effect model was adopted. Publication bias was checked by Begg funnel plots. Statistical significance was set at $p<0.05$.

\section{Results}

\section{Study selection and characteristics}

A total of 306 studies that examined the correlation of GP73 and HCC were identified by using the above keywords. Thirty-five studies were excluded because they were case reports, reviews, letters to the editor, and duplications. After the full text was reviewed and evaluated, 46 studies were removed because of a lack of relevant topic or sufficient survival data. Finally, nine studies with 1292 patients met the requirements and were used to evaluate the prognostic value of GP73 (Figure 1) [15, 17, 19, 20, 23-27]. All studies included in the meta-anal- 
ysis used immunohistochemistry to detect GP73 expression levels. OS can be extracted from all articles, while DFS was presented in 3 studies. The quality assessment of articles, as evaluated by the Newcastle-Ottawa scale, ranged from 5 to 8 (with a mean of 6.4). The clinicopathological characteristics of the nine studies are summarized in Table I.

\section{GP73 expression and OS in HCC}

Nine articles reported the association of GP73 expression and OS in HCC [15, 17, 19, 20, 23-27]. The pooled data demonstrated a significant correlation between high GP73 expression and poor OS $(\mathrm{HR}=1.76,95 \% \mathrm{Cl}: 1.35-2.31, p<0.0001$; Figure 2$)$. A random effects model was adopted in this metaanalysis as there was obvious heterogeneity in the data $\left(I^{2}=56 \%, p=0.02\right)$.

\section{GP73 expression and DFS in HCC}

The association of GP73 with DFS was presented in three studies [20, 24, 25]. The overall meta-analysis showed no significant association between elevated GP73 levels and DFS (HR $=1.19$, $95 \% \mathrm{Cl}: 0.57-2.52, p=0.64$; Figure 3$)$. Since heterogeneity is obvious in the study $\left(I^{2}=81 \%, p=\right.$ $0.005)$, the random effects model was applied to calculate the pooled HR.

\section{GP73 expression and clinicopathological characteristics of HCC}

Several articles assessed the correlation of high GP73 expression with HBV or HCV infection, tu- mour size, histological grade, vascular invasion, liver cirrhosis, tumour number, and tumour stage. The result indicated that high GP73 expression was significantly correlated with higher tumour grade $(\mathrm{OR}=3.72,95 \% \mathrm{Cl}: 1.26-11.02, p=0.02)$ and later tumour stage $(\mathrm{OR}=3.43,95 \% \mathrm{Cl}: 1.74-$ 6.73, $p=0.0003$ ) (Table II).

\section{Subgroup analysis}

We also performed subgroup analyses to investigate the association of GP73 expression and OS according to sample size and follow-up period (Table III). In the results of the subgroup analysis of the studies by sample size, the HR obtained from studies with more than 90 cases was 1.84 (95\% Cl: 1.54-2.19, $p<0.001)$. However, the pooled HR was 1.47 (95\% Cl: $0.85-2.51, p=0.17$ ) in the studies with fewer than 90 cases. Similarly, when the studies were analysed based on the follow-up period, the results showed that there was a significantly poorer prognosis among studies with longer follow-up periods ( $\geq 60$ months) $(\mathrm{HR}=$ 1.85, 95\% Cl: 1.49-2.30, $p<0.001)$. In the combined data from articles with a shorter follow-up period (< 60 months), there was no significant correlation between high GP73 expression and OS $(\mathrm{HR}=1.51,95 \% \mathrm{Cl}: 0.83-2.78, p=0.18)$.

\section{Publication bias}

The function of publication bias analysis is to assess the reliability of the meta-analysis results, especially those with statistical significance [28].
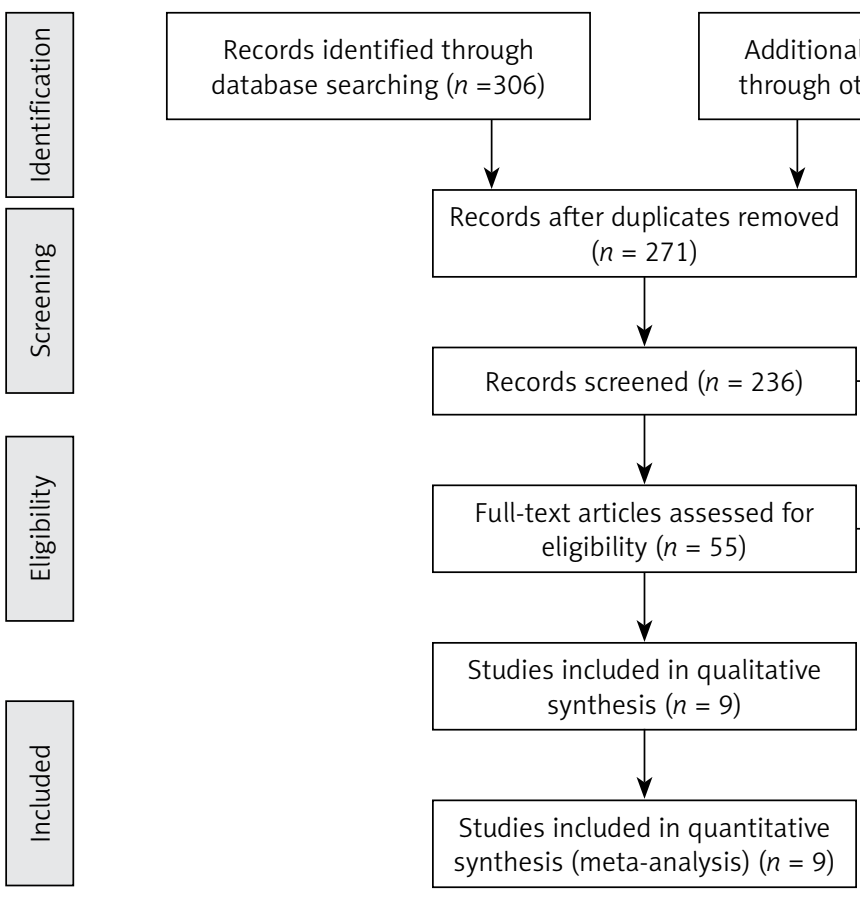

Additional records identified through other sources $(n=0)$

Figure 1 . Flow diagram of the study selection process 
The Begg funnel plot method was applied to test publication bias in this meta-analysis. We observed no publication bias for the outcomes of OS, with no asymmetry observed in the funnel plot (Figure 4).

\section{Discussion}

The HCC, a malignant tumour, accounts for nearly half of all cancer cases in China. Due to the high rate of recurrence and metastasis, patients present poor prognosis after resection [22, 29]. It is urgent to discover molecular markers that can predict the recurrence and metastasis of HCC. The most recent studies showed that GP73 expression was associated with invasion and metastasis in HCC [15-17]. Nevertheless, the conclusions from previous studies were inconsistent. Therefore, it is appropriate to conduct a meta-analysis to systematically evaluate the association of GP73 expression and HCC prognosis.

In the present study, 9 studies were included in the meta-analysis. The number of studies included in the meta-analysis was nearly double that in Hou's study. Therefore, the results from the metaanalysis are more comprehensive and reliable. We first evaluated the relationship of high GP73 expression and survival outcomes. The combined data suggested that overexpression of GP73 was associated with poorer OS for patients with HCC $(p<0.0001)$. Patients with overexpression of GP73 were vulnerable to shorter OS than those with low GP73 expression. Furthermore, combined HR for OS was lower than that in the Hou et al. meta-analysis. The main reason is that more studies were included in this meta-analysis than were included in the Hou et al. study, which may have produced more reliable and powerful statistics. However, the pooled HR for DFS was not significant. The reasons may be as follows: first, only three of the seven articles included in the metaanalysis demonstrated a correlation between high GP73 expression and DFS. Further studies are required to better test the results. Second, patients included in the meta-analysis all originated from China, which increases regional limitations. Therefore, the DFS outcomes need to be verified by collecting more data from more regions in prospective studies. Furthermore, we also proved that overexpression of GP73 was tightly associated with higher tumour grade and later tumour stage. The results were in accord with those from studies by Riener et al. [30] and Shimada et al. [31], which showed that these clinicopathological features are highly correlated with the aggression and metastasis of HCC.

Heterogeneity is obvious in the meta-analysis for OS and DFS. Stratified subgroup analyses were applied to decrease the heterogeneity. When we

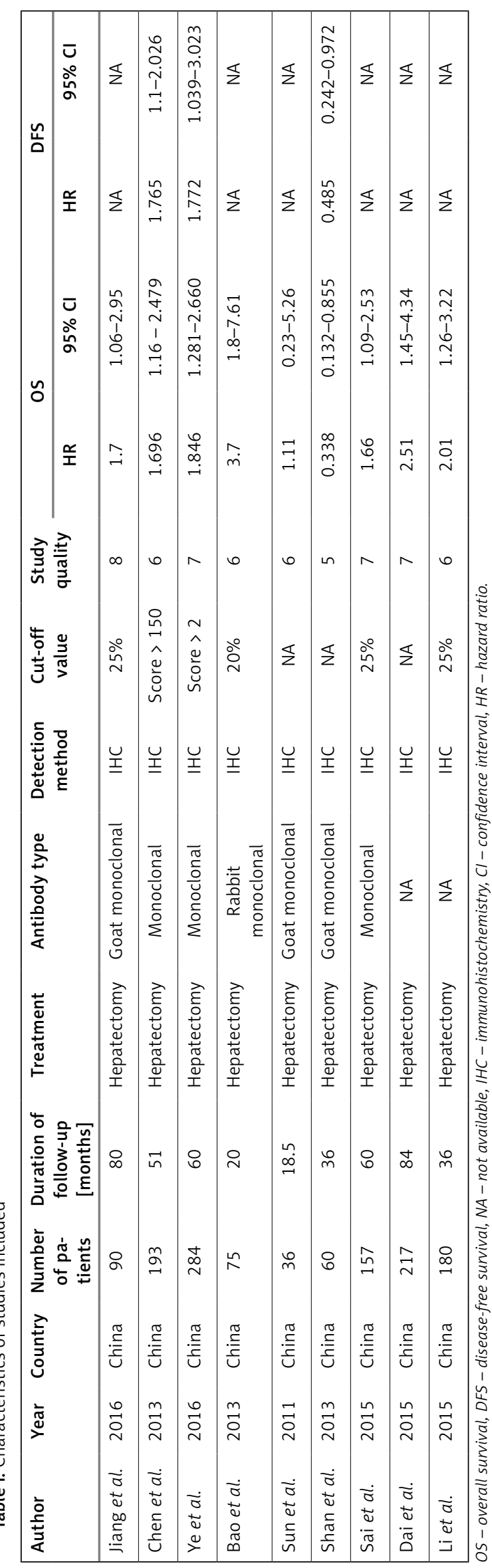




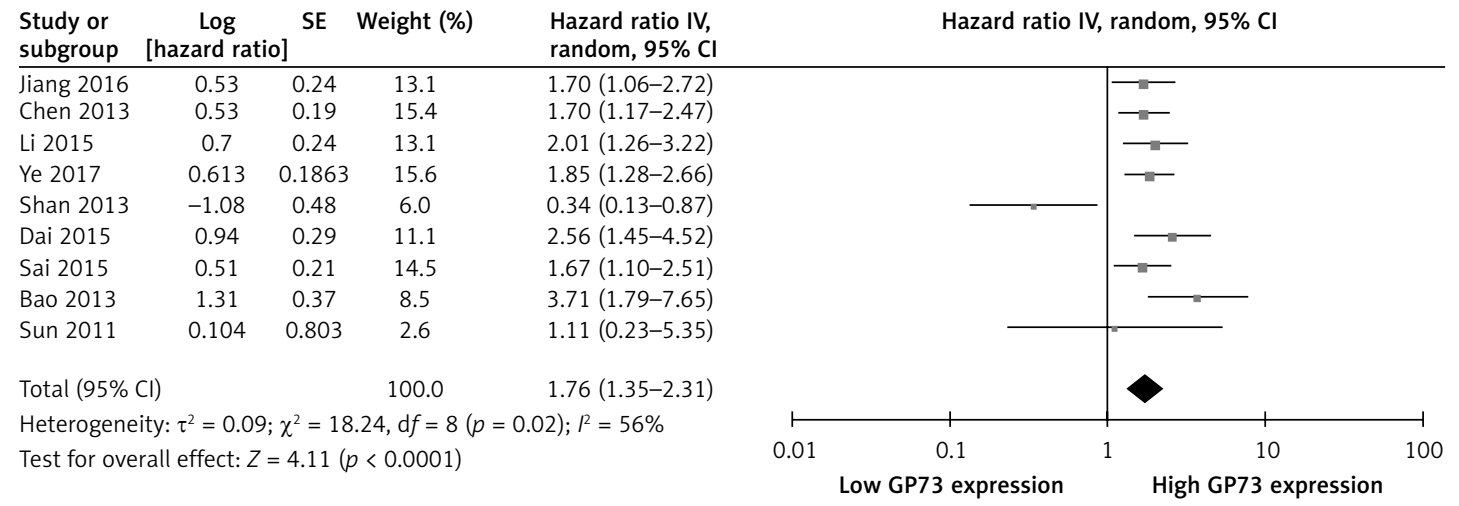

Figure 2. Association between GP73 overexpression and overall survival of HCC

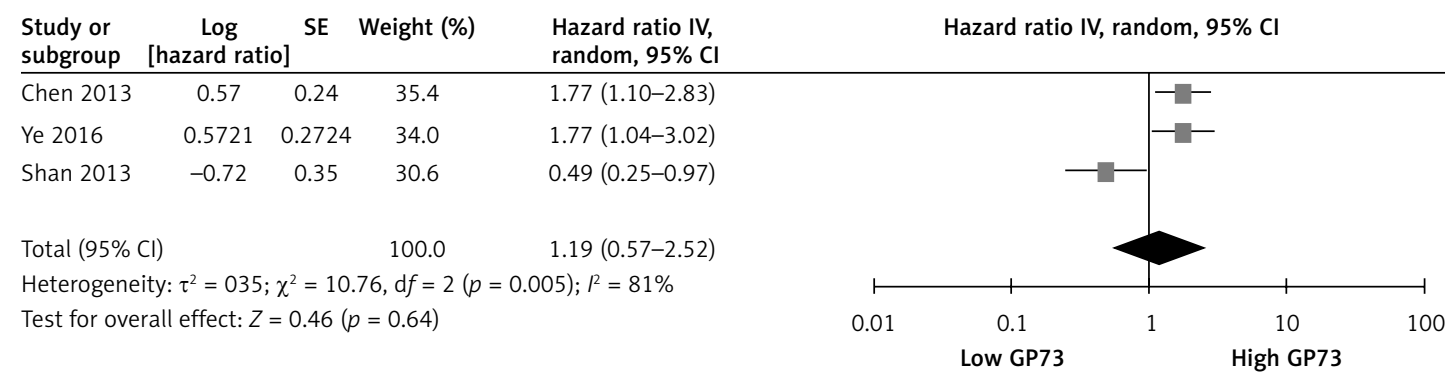

Figure 3. Association between GP73 overexpression and disease-free survival of HCC

Table II. Association between high GP73 expression and clinicopathological features

\begin{tabular}{|c|c|c|c|c|c|c|c|}
\hline \multirow[t]{2}{*}{ Parameter } & \multirow{2}{*}{$\begin{array}{c}N \text { of } \\
\text { studies }\end{array}$} & \multirow{2}{*}{$\begin{array}{c}N \text { of } \\
\text { patients }\end{array}$} & \multirow{2}{*}{$\begin{array}{l}\text { Effect } \\
\text { model }\end{array}$} & \multirow[t]{2}{*}{ OR $(95 \% \mathrm{Cl})$} & \multirow[t]{2}{*}{$P$-value } & \multicolumn{2}{|c|}{ Heterogeneity test } \\
\hline & & & & & & $I^{2}(\%)$ & $P$-value \\
\hline $\mathrm{HBV}(+/-)$ & 5 & 829 & Fixed & $0.95(0.65-1.40)$ & 0.80 & 0 & 0.52 \\
\hline $\mathrm{HCV}(+/-)$ & 2 & 253 & Fixed & $0.87(0.48-1.57)$ & 0.64 & 0 & 0.84 \\
\hline Tumour size $(\geq 5 \mathrm{~cm} /<5 \mathrm{~cm})$ & 5 & 934 & Fixed & $0.99(0.63-1.58)$ & 0.97 & 0 & 0.66 \\
\hline Histological grade (G2-3/G1) & 5 & 816 & Random & $3.72(1.26-11.02)$ & 0.02 & 90 & $<0.001$ \\
\hline $\begin{array}{l}\text { Vascular invasion (positive/ } \\
\text { negative) }\end{array}$ & 5 & 1009 & Random & $1.29(0.58-2.89)$ & 0.82 & 83 & $<0.001$ \\
\hline Liver cirrhosis & 6 & 1091 & Random & $1.28(0.75-2.19)$ & 0.36 & 78 & $<0.001$ \\
\hline $\begin{array}{l}\text { Tumour number (multiple/ } \\
\text { single) }\end{array}$ & 5 & 874 & Random & $1.68(0.51-5.54)$ & 0.4 & 86 & $<0.001$ \\
\hline Stage (III-IV/II-II) & 6 & 986 & Random & $3.43(1.74-6.73)$ & 0.0003 & 67 & 0.01 \\
\hline
\end{tabular}

$\mathrm{Cl}$ - confidence interval, GP73 - Golgi protein 73, HBV - hepatitis B virus, $H C V$ - hepatitis C virus, $N$ - number, $O R$ - odds ratio.

Table III. Subgroup analyses for effect of GP73 on HCC overall survival

\begin{tabular}{|c|c|c|c|c|c|c|}
\hline \multirow[t]{2}{*}{ Parameter } & \multirow[t]{2}{*}{$N$ of studies } & \multirow[t]{2}{*}{ Effect model } & \multirow[t]{2}{*}{ HR $(95 \% \mathrm{Cl})$} & \multirow[t]{2}{*}{$P$-value } & \multicolumn{2}{|c|}{ Heterogeneity test } \\
\hline & & & & & $I^{2}(\%)$ & $P$-value \\
\hline Overall & 9 & Random & $1.61(1.15-2.27)$ & 0.006 & 63 & 0.01 \\
\hline \multicolumn{7}{|c|}{ Sample size: } \\
\hline$<90$ & 3 & Random & $1.47(0.85-2.51)$ & 0.17 & 87 & $<0.001$ \\
\hline$\geq 90$ & 6 & Fixed & $1.84(1.54-2.19)$ & $<0.001$ & 0 & 0.86 \\
\hline \multicolumn{7}{|c|}{ Duration of follow-up [months]: } \\
\hline$<60$ & 5 & Random & $1.51(0.83-2.78)$ & 0.18 & 76 & 0.002 \\
\hline$\geq 60$ & 4 & Fixed & $1.85(1.49-2.30)$ & $<0.001$ & 0 & 0.65 \\
\hline
\end{tabular}

$\mathrm{Cl}$ - confidence interval, GP73 - Golgi protein 73, HCC - hepatocellular carcinoma, HR - hazard ratio, N- number. 


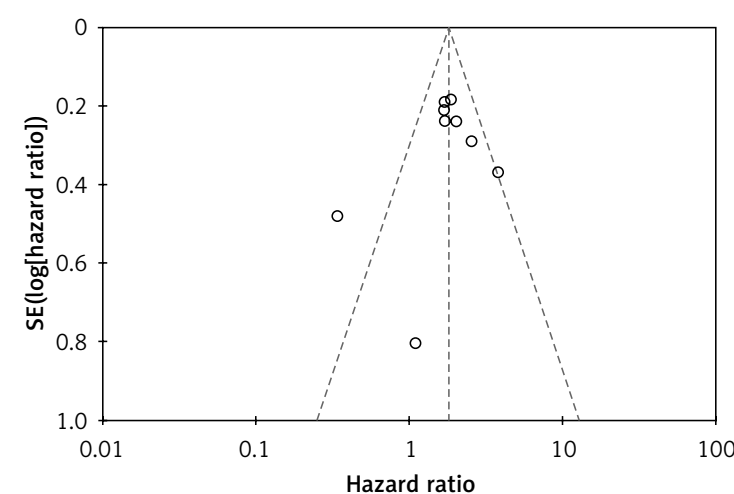

Figure 4. Funnel plot for assessing publication bias in OS

divided all the literature into two subgroups according to sample size, a significant correlation of GP73 overexpression with poorer OS was presented for studies with more than 90 cases, while no association was found in the studies with fewer than 90 cases. Therefore, more studies are required to determine the reliable value of GP73 for predicting OS in HCC. Similarly, a significant correlation was only observed in studies with a follow-up period $\geq 60$ months, suggesting that the status of GP73 expression was more suitable for evaluating long-term outcomes in HCC.

We must acknowledge that several limitations exist in this meta-analysis. First, if the studies included in the meta-analysis did not provide HRs and their $95 \% \mathrm{Cls}$ directly, then the data were extrapolated indirectly using the available data or Kaplan-Meier survival curve, which may result in imprecise outcomes. Second, the patients in the studies included in the meta-analysis had different ages, cut-off values, and follow-up times. Furthermore, all patients included in the meta-analysis originated from China. Third, publication bias cannot be ignored, because studies with positive results tend to be published more often than those with negative outcomes, which will exaggerate the correlation of high GP73 expression and poor prognosis.

In conclusion, despite the limitations mentioned above, the meta-analysis indicated that high GP73 expression correlated with poor prognosis in HCC. In addition, our study showed that GP73 overexpression was associated with later tumour stage and higher tumour grade in HCC, which indicated that GP73 may play a vital role in tumour invasion and progression. Furthermore, more prospective clinical trials are needed to evaluate the correlation of GP73 expression with prognosis in HCC.

\section{Acknowledgments}

This study was supported by the National Major Scientific and Technological Project during the Twelfth Five-year Plan Period (No. 2014ZX 10005002-002), the National Major Scientific and Technological Project during the Thirteenth Fiveyear Plan Period (No. 2017ZX10205501-001-002) and the Special Research Project of the Traditional Chinese Medicine Industry (No. 201507005).

\section{Conflict of interest}

The authors declare no conflict of interest.

\section{References}

1. Ferlay J, Shin HR, Bray F, Forman D, Mathers C, Parkin DM. Estimates of worldwide burden of cancer in 2008: GLOBOCAN 2008. Int J Cancer 2010; 127: 2893-917.

2. Bruix J, Llovet JM. Prognostic prediction and treatment strategy in hepatocellular carcinoma. Hepatology 2002; 35: 519-24.

3. Shiraha H, Yamamoto K, Namba M. Human hepatocyte carcinogenesis. Int J Oncol 2013; 42: 1133-8.

4. Chen G, Li X, Yang J, et al. Prognostic significance of cyclooxygenase- 2 expression in patients with hepatocellular carcinoma: a meta-analysis. Arch Med Sci 2016; 12: 1110-7.

5. El-Serag HB, Rudolph KL. Hepatocellular carcinoma: epidemiology and molecular carcinogenesis. Gastroenterology 2007; 132: 2557-76.

6. Lei Z, Wen T, Xu M, et al. Sorafenib combined with hepatectomy in patients with intermediate-stage and advanced hepatocellular carcinoma. Arch Med Sci 2017; 13: 1383-93.

7. Kladney RD, Bulla GA, Guo L, et al. GP73, a novel Golgi-localized protein upregulated by viral infection. Gene 2000; 249: 53-65.

8. Parkin DM. Global cancer statistics in the year 2000. Lancet Oncol 2001; 2: 533-43.

9. Kandil DH, Cooper K. Glypican-3: a novel diagnostic marker for hepatocellular carcinoma and more. Adv Anat Pathol 2009; 16: 125-9.

10. Libbrecht L, Severi T, Cassiman D, et al. Glypican-3 expression distinguishes small hepatocellular carcinomas from cirrhosis, dysplastic nodules, and focal nodular hyperplasia-like nodules. Am J Surg Pathol 2006; 30: 1405-11.

11. Liu H, Li PY, Qu CF, et al. Diagnostic value of glypican-3 in serum and liver for primary hepatocellular carcinoma. World J Gastroenterol 2010; 16: 4410-5.

12. Marrero JA, Romano PR, Nikolaeva O, et al. GP73, a resident Golgi glycoprotein, is a novel serum marker for hepatocellular carcinoma. J Hepatol 2005; 43: 1007-12.

13. Hu JS, Wu DW, Liang S, Miao XY. GP73, a resident Golgi glycoprotein, is sensibility and specificity for hepatocellular carcinoma of diagnosis in a hepatitis B-endemic Asian population. Med Oncol 2010; 27: 339-45.

14. Xu WF, Fei YM, Zhou JK, et al. Significance of serum Golgi protein 73 (GP73), alpha-fetoprotein (AFP) and lectin-reactive alpha-fetoprotein (AFP-L3) expresssion in primary hepatic carcinoma. Zhonghua Shi Yan He Lin Chuang Bing Du Xue Za Zhi 2011; 25: 286-8.

15. Sun Y, Yang H, Mao Y, et al. Increased Golgi protein 73 expression in hepatocellular carcinoma tissue correlates with tumor aggression but not survival. J Gastroenterol Hepatol 2011; 26: 1207-12.

16. Mao Y, Yang H, Xu H, et al. Golgi protein 73 (GOLPH2) is a valuable serum marker for hepatocellular carcinoma. Gut 2010; 59: 1687-93. 
17. Jiang K, Li W, Shang S, et al. Aberrant expression of Golgi protein 703 is indicative of a poor outcome in hepatocellular carcinoma. Oncol Rep 2016; 35: 2141-50.

18. Hou X, Yang L, Jiang X, Li X, Liu J. Prognostic significance of Golgi protein 73 in hepatocellular carcinoma after hepatectomy in the Chinese population: a meta-analysis. Int J Clin Exp Med 2017; 10: 48-58.

19. Wenli S, Wang L, Zheng W, et al. Abnormal expression of Golgi protein 73 in clinical values and their role in HBV-related hepatocellular carcinoma diagnosis and prognosis. Hepatitis Monthly 2015; 15: e32918.

20. Ye QH, Zhu WW, Zhang JB, et al. GOLM1 modulates EGFR/RTK cell-surface recycling to drive hepatocellular carcinoma metastasis. Cancer Cell 2016; 30: 444-58.

21. Tierney JF, Stewart LA, Ghersi D, Burdett S, Sydes MR. Practical methods for incorporating summary time-toevent data into meta-analysis. Trials 2007; 8: 16.

22. Fu SJ, Qi CY, Xiao WK, Li SQ, Peng BG, Liang LJ. Glypican-3 is a potential prognostic biomarker for hepatocellular carcinoma after curative resection. Surgery 2013; 154: 536-44.

23. Bao YX, Cao Q Yang Y, et al. Expression and prognostic significance of golgiglycoprotein73 (GP73) with epithelial-mesenchymal transition (EMT) related molecules in hepatocellular carcinoma (HCC). Diagn Pathol 2013; 8: 197.

24. Chen MH, Jan YH, Chang MH, et al. Expression of GOLM1 correlates with prognosis in human hepatocellular carcinoma. Ann Surg Oncol 2013; 20: 616-24.

25. Shan SG, Gao YT, Xu YJ, et al. Gradually increased Golgi protein 73 expression in the progression of benign liver diseases to precancerous lesions and hepatocellular carcinoma correlates with prognosis of patients. Hepatol Res 2013; 43: 1199-210.

26. Dai T, Zhang D, Cai M, et al. Golgi phosphoprotein 3 (GOLPH3) promotes hepatocellular carcinoma cell aggressiveness by activating the NF-kappaB pathway. J Pathol 2015; 235: 490-501.

27. Li Q, Ma Y, Xu W. High GOLPH3 expression is associated with poor prognosis and invasion of hepatocellular carcinoma. Mol Med Rep 2015; 11: 4315-20.

28. Y E, He N, Wang Y, Fan H. Percutaneous transluminal angioplasty (PTA) alone versus PTA with balloon-expandable stent placement for short-segment femoropopliteal artery disease: a metaanalysis of randomized trials. J Vasc Interv Radiol 2008; 19: 499-503.

29. Jing H, Cheng W, Zhang JW, Han X, Shao H, Sun YX. Galactosylated poly-L-lysine targeted microbubbles for ultrasound mediated antisense c-myc gene transfection in hepatocellular carcinoma cells. Arch Med Sci 2015; 11: 292-300.

30. Riener MO, Stenner F, Liewen H, et al. Golgi phosphoprotein 2 (GOLPH2) expression in liver tumors and its value as a serum marker in hepatocellular carcinomas. Hepatology 2009; 49: 1602-9.

31. Shimada K, Sano T, Sakamoto Y, Kosuge T. A long-term follow-up and management study of hepatocellular carcinoma patients surviving for 10 years or longer after curative hepatectomy. Cancer 2005; 104: 1939-47. 